\title{
BMJ Global Health Estimating the incidence of abortion: a comparison of five approaches in Ghana
}

\author{
Sarah C Keogh, ${ }^{1}$ Easmon Otupiri, ${ }^{2}$ Doris W Chiu, ${ }^{1}$ Chelsea B Polis (D) , ${ }^{1,3}$ \\ Rubina Hussain, ${ }^{1}$ Suzanne O Bell, ${ }^{4}$ Emmanuel K Nakua, ${ }^{5}$ \\ Roderick Larsen-Reindorf ${ }^{2}$
}

To cite: Keogh SC, Otupiri E, Chiu DW, et al. Estimating the incidence of abortion: a comparison of five approaches in Ghana. BMJ Global Health 2020;5:e002129. doi:10.1136/ bmjgh-2019-002129

\section{Handling editor Sanni Yaya}

- Additional material is published online only. To view please visit the journal online (http://dx.doi.org/10.1136/ bmjgh-2019-002129).

Received 30 0ctober 2019 Revised 21 February 2020 Accepted 11 March 2020
Check for updates

\section{Author(s) (or their} employer(s)) 2020. Re-use permitted under CC BY-NC. No commercial re-use. See rights and permissions. Published by BMJ.

For numbered affiliations see end of article.

Correspondence to Dr Sarah C Keogh; skeogh@guttmacher.org

\section{ABSTRACT}

Introduction Induced abortion estimates are critical for reproductive health programming. In countries like Ghana where abortion is somewhat legally restricted and highly stigmatised, official records are incomplete and different approaches are needed to measure abortion incidence. We conducted a study in Ghana to test five methodologies for estimating incidence: direct reporting, the list experiment, the confidante method, the Abortion Incidence Complications Method (AICM) and a modified AICM. Methods The direct reporting, list experiment and confidante method were implemented through a nationally representative community-based survey (CBS) of 4722 women. The AICM used data from a nationally representative health facilities survey (HFS) and a knowledgeable informant survey. The modified AICM combined CBS and HFS data. For each approach, we calculated abortion incidence nationally and for Ghana's three ecological zones and conducted checks to determine the most internally valid approaches.

Results National incidence estimates ranged from 27 per 1000 (AICM) to 61 (confidante method). The Northern zone displayed lower rates than the other two zones for all approaches. Validity and reliability checks found that the list experiment was invalid. The approaches that stood up to the internal validity checks and were most reliable were the direct reporting, confidante method and modified AICM. These approaches provide lower and upper bound estimates for the abortion rate, and the mean of the estimates from the three approaches yields a final abortion rate of 44 per 1000 and an unintended pregnancy rate of 103 per 1000 .

Conclusions Comparing five approaches to estimating abortion enabled cross-validation of findings and highlighted strengths, pitfalls and requirements of each approach that can inform abortion estimation in other settings.

\section{INTRODUCTION}

Estimating induced abortion incidence is critical for designing appropriate reproductive health policies and programmes and measuring other outcomes such as unintended pregnancy rates. Yet in countries where induced abortion is legally restricted or highly stigmatised, reliable estimates of

\section{Key questions}

What is already known?

- The two most common approaches to estimating abortion (direct questioning and the Abortion Incidence Complications Method (AICM)) suffer from under-reporting and heavy reliance on expert opinions, respectively.

- The List Experiment has yielded mixed results, whereas network approaches such as the Best Friend approach and the Anonymous Third-Party Reporting have yielded promising results.

What are the new findings?

- This is the first study to test more than four approaches in one population, including two newly proposed methodologies (the modified AICM and the confidante method).

- By cross-validating components of the estimates against each other, we identified the most internally valid approaches (modified AICM, direct reporting and confidante method), which together yielded a national abortion incidence rate of 44 per 1000 and a pregnancy rate of 194 per 1000 .

\section{What do the new findings imply?}

- Implementing the confidante method as a precursor to direct questioning may help increase self-reporting, regardless of the reliability of the network-based estimate.

- If reliable postabortion care caseloads can be collected alongside community-based survey data then the modified AICM can provide a more robust estimate than methodologies based on experts opinions but is only recommended if abortion stigma makes direct reporting and network-based approaches unreliable.

- The approach yielding the most robust and internally valid estimates will likely vary according to the cultural and legal context, the information women share among themselves, literacy and numeracy, and abortion stigma.

abortion incidence are often lacking. ${ }^{1}$ In Ghana, although abortion is legal under several circumstances, many women are unaware of the law and obtain clandestine abortions. $^{2}$ Thus, many abortions are not 
captured in official records, necessitating other measurement approaches. We conducted a study in Ghana testing the five methodologies we thought to be most promising for estimating abortion incidence in countries where abortion is either legally restricted or highly stigmatised. $^{3}$

One common approach, used previously in Ghana, ${ }^{4-7}$ directly asks women if they have had an abortion. Given the reluctance to disclose abortion, direct reporting underestimates incidence. ${ }^{8}$ However, it provides a useful minimum estimate, and we therefore included it in this study (approach \#1). Variations of direct reporting that preserve anonymity ${ }^{9}{ }^{10}$ have not been shown to consistently outperform face-to-face questionnaires ${ }^{911}$ and are unsuitable for low-literacy populations.

The Abortion Incidence Complications Method $(\mathrm{AICM})^{12}$ (approach \#2) is an indirect approach that aims to circumvent the under-reporting inherent in direct reporting; it is the prevailing approach in abortion incidence estimation. In the standard AICM, researchers estimate the number of abortions as the number of women receiving postabortion care (PAC) in a nationally representative survey of health facilities, multiplied by an adjustment factor, or multiplier, that accounts for all the abortions that do not lead to facility-based treatment (obtained from a survey of knowledgeable informants). ${ }^{13}$ Due to the complexity of the AICM when applied to Ghana, we describe the methodology and results in a separate paper. $^{2}$ The AICM has limitations, ${ }^{3} 12$ including reliance on expert opinion to estimate the proportion of abortions receiving treatment. To address this, we propose a modified version of the AICM (approach \#3), in which we calculate the multiplier using information collected from women directly. ${ }^{3}$

The List Experiment, which investigators have used to estimate abortion in Liberia $^{14}$ and Rajasthan, ${ }^{15}$ involves presenting a list of non-sensitive events and asking respondents how many (but not which ones) they have experienced. In half of the sample (the treatment group), abortion is added to the list. Abortion incidence is the difference between the mean number of events in each list. To increase power and minimise list effects, the Double List Experiment (approach \#4) ${ }^{14} 16$ includes two lists (A and $\mathrm{B}$ ); each respondent receives the treatment version of one list and the control version of the other. ${ }^{3} \mathrm{~A}$ recent commentary reviewed considerations relevant to the List Experiment for abortion estimation ${ }^{17}$ and while the approach has yielded mixed results, ${ }^{14} 15$ we determined its strengths ${ }^{318}$ justified inclusion in this study.

Finally, social network-based approaches ask respondents about abortions among their female friends and relatives based on the assumption that women are less likely to under-report others' abortions. ${ }^{19-22}$ We propose the confidante method (approach \#5) in which respondents are asked questions about up to three of their closest female confidantes, blending the Best Friend approach $^{20}$ and the Anonymous Third Party Reporting ${ }^{19}$ to capitalise on the strengths of each. ${ }^{3}$
Other approaches to estimating abortion incidence have been proposed, but have yielded inconsistent results. ${ }^{11}$ We selected the five approaches compared in this paper because we deemed them to be the most promising for low-resource settings with unreliable official statistics. ${ }^{3}$ Including the two prevailing approaches to measuring abortion (direct reporting, AICM) enables assessment of how more novel approaches compare. More details on the strengths and weaknesses of each approach and considerations around validity and precision are available elsewhere. ${ }^{3}$ A small number of studies have compared multiple methods for estimating abortion incidence ${ }^{915182324}$; this is the first to test over four approaches, including both female survey-based and indirect facility-based methodologies, and to propose two new approaches.

\section{METHODS \\ Data collection}

The List Experiment, confidante method and direct reporting use data from a nationally representative community-based survey (CBS) of women. The AICM uses data from two surveys: a nationally representative health facilities survey (HFS) collecting annual caseloads of PAC and induced abortions; and a knowledgeable informant survey (KIS) estimating the proportion of abortions treated for complications in facilities. The modified AICM uses data from the HFS and CBS.

We describe the HFS and KIS elsewhere. ${ }^{2}$ We drew the CBS sample using a two-stage stratified cluster design with probability proportional to size sampling. First, 100 enumeration areas (EAs) were selected, stratified by urban/rural residence and region. Following household mapping, we randomly selected 42 households from each EA and conducted household surveys to gather socioeconomic information and identify eligible female respondents (aged 15-49 years) whom we invited to participate in the CBS. On obtaining informed consent, a trained interviewer administered a structured questionnaire faceto-face using an Android smartphone enabled with the Open Data Kit electronic data collection software, in a private place. Interviewers lived locally and were familiar with the language and culture. We conducted interviews in English or the respondent's local language. As there are 49 official languages in Ghana (many unwritten), survey questions were translated orally by the interviewer using translations agreed on during interviewer training.

Kwame Nkrumah University of Science and Technology (KNUST) conducted the fieldwork between May and July 2018, with technical support from the Guttmacher Institute and the Performance Monitoring and Accountability team at the Johns Hopkins Bloomberg School of Public Health. Ethical approval was obtained from the Institutional Review Boards of the Guttmacher Institute (IRB00002197) and the Johns Hopkins Bloomberg School of Public Health (00008463), and from the 
KNUST Committee on Human Research, Publication and Ethics (CHRPE/AP/210/18).

To improve the validity of responses, we placed abortion-related modules in a particular order, starting with the List Experiment (so respondents did not know which list events we were interested in), followed by the confidante method (because talking about friends' abortions may encourage later self-disclosure of abortion), and ending with direct reports. Below, we describe how each of the five estimation approaches was implemented.

\section{List Experiment}

For the Double List Experiment, we created two lists (A and B) of four non-sensitive events related to health or reproduction (eg, taking an ambulance, having a period) and asked respondents how many of these events they had experienced in the last 12 months. We randomly allocated respondents to two equal-sized groups ( 1 and 2). We added abortion as a fifth item to list A for group 1 and list B for group 2. Group 1 received list A with the abortion item, followed by list B without abortion, whereas group 2 received list $\mathrm{B}$ with abortion followed by list A without abortion. To validate this approach, we also included a practice list that asked about a non-sensitive event (having eaten koose, a local food, in the past week), and compared the estimate derived from the practice list with the estimate from a direct question at the end of the questionnaire.

\section{Confidante method}

We asked each respondent to think about up to three confidantes aged 15-49 years who share private and confidential information with her and with whom she also shares such information. We collected information on each confidante's sociodemographic characteristics, contraceptive use, abortion experience (and timing) within the past 5 years, existence of complications and receipt of treatment. One of the key assumptions for the confidante method is that respondents are selecting confidantes independently of the confidante's likelihood of having had an abortion. To minimise the risk of respondents' thinking about abortion when selecting their confidantes, we placed the confidante module before direct questions about abortion and ensured that the confidante-generating question did not mention abortion. Nonetheless, the module had to be placed after the List Experiment, and our Institutional Review Board required inclusion of the term 'abortion' in the consent form, so respondents may have been primed to think about confidantes who had an abortion. To help determine whether this was the case, we conducted a phone follow-up of 406 randomly selected respondents to enquire about whether they were thinking about abortion when selecting confidantes.

\section{Direct reporting}

Respondents were asked whether they had abortions in the past 5 years and the timing of each abortion.
Additionally, some women may take action to bring back a late period without necessarily knowing whether they are pregnant or considering it an abortion. To test whether self-reporting could be improved by including these events, before the direct question about abortion, we asked respondents if they had done anything to bring back a late period and classified these events as abortions if the respondent believed that she was pregnant at the time and if her period was at least 7 days late. We call these events menstrual regulations (MR) for short.

\section{AICM and modified AICM}

Data collection for the standard AICM is described in Polis $e t a l^{2}$ For the modified AICM, we estimated PAC caseloads from the HFS (as in the standard AICM) but estimated the multiplier from women's self-reported abortions (instead of the KIS). We asked women who reported an abortion in the past 3 years whether they had experienced complications and if so, whether and where they had sought treatment. This enabled us to calculate the proportion of all abortions that resulted in complications treated in a health facility (the inverse of the multiplier).

\section{Patient and public involvement}

We did not directly involve identified abortion patients in study planning, but we sought guidance for study planning and results dissemination from our Technical Advisory Committee, which included community representatives and technical experts.

\section{Data analysis}

The project and analysis plans were pre-registered on the Open Science Framework (https://osf.io/285ew). We used Stata 15 for data cleaning and analysis. We used Stata's syyset command to account for the complex survey design of the CBS. Although the CBS sample was drawn to be nationally and zonally representative, the weighted sample characteristics differed significantly on zone and union status from the 2017 Ghana Maternal Health Survey (GMHS) population distributions (which we viewed as more accurate due to the substantially larger sample size) (online supplementary appendix A). To ensure the CBS was representative, we used the GMHS to produce poststratification weights. We similarly weighted the confidante data to ensure that it was representative and accounted for clustering by the respondent. Estimates of abortion incidence (number of abortions per 1000 women aged 15-49 per year) from each methodology are computed nationally and for each of Ghana's three ecological zones (Coastal, Middle and Northern). For each rate, we calculate $95 \%$ CIs. We then use these CIs to determine whether the rates from the different approaches differ significantly or not: if the CIs from two approaches do not overlap, the rates are significantly different. ${ }^{25}$

For direct reporting and the confidante method, we first calculated annual abortion rates for each of the past 


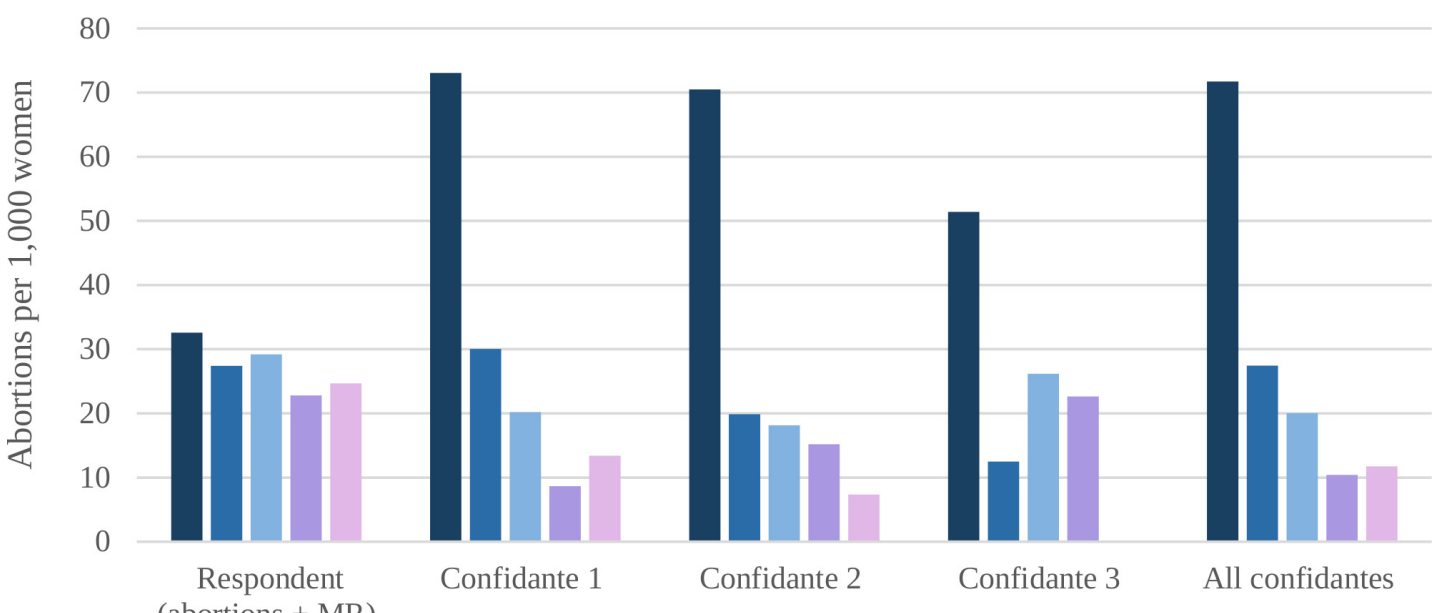
(abortions + MR)

Past year $\quad$ 1-2 years ago

2-3 years ago

- 3-4 years ago

4-5 years ago

Figure 1 MR or action to bring back a late period without necessarily knowing whether one is pregnant. 'All confidantes' estimates were calculated by pooling all confidantes, giving equal weight to each. MR, menstrual regulation.

5 years to check for potential telescoping (ie, temporal displacement) or attrition (in knowledge of confidantes' abortions or reporting) over time (figure 1). As we do not expect any secular changes in abortion rates over the past 5 years, a higher rate in the past year followed by a sudden drop in the second year would indicate telescoping of abortion events to the past year, whereas gradual decreases in rates from year 2 to 5 would indicate attrition in reporting. Both these patterns can be observed in figure 1. Given considerable telescoping to the past year and some attrition over time in both approaches, we present direct report and confidante estimates as annualised rates over the past 3 years (mid-2015 to mid-2018). Estimates for the AICM and the modified AICM are for the year 2017 (the timeframe for which the HFS caseloads were collected), and estimates for the List Experiment are for the past year (mid-2017 to mid-2018). As we do not expect secular changes in abortion rates between these overlapping periods, these slight differences in time frames should not hinder the comparison of rates from the different approaches.

For the confidante estimates, we made two adjustments to counteract potential biases from 'missing' confidantes who may have different abortion behaviours, and nondisclosure of some confidantes' abortions to the respondent (transmission bias; see table 1 for more details).

We compared the five estimates with respect to compliance with assumptions, consistency across subgroups, reliability and precision. ${ }^{3}$ We also estimated zonal and national numbers of unintended pregnancies; see the online supplementary appendix B for methodological details.

\section{RESULTS}

The List Experiment yielded two very different national estimates for each list: 14 per 1000 (list A; table 2) vs 57 (list B). The lack of consistency between the two rates, and their wide $95 \%$ CIs suggest the estimates are unreliable. Moreover, the Northern zone had a negative rate of -2 per 1000 , which is invalid. The practice list measuring the incidence of eating koose in the past week did not yield accurate results either as it gave an estimate of 29\%, whereas the 'true' prevalence obtained from the direct question was $43 \%$, and CIs for the two estimates did not overlap, making the estimates significantly different (data not shown). These findings suggest the list experiment (as implemented in both the practice list and the real list) was poorly understood and calls into question its validity in this setting. Therefore, we exclude it from subsequent comparisons.

The 3-year annualised rate from the confidante approach (adjusted for the 'missing' confidantes but not for transmission bias) was 38 per 1000 nationally (95\% CI: 35 to 41), ranging from 18 (Northern zone) to 43 (Coastal zone; table 1). Adjusting for transmission bias resulted in a national rate of 61 per 1000, ranging from 34 (Northern zone) to 71 (Coastal zone).

The 3-year annualised abortion incidence rate from direct reporting on abortion (including menstrual regulation (MR)) was 30 per 1000 nationally (95\% CI: 24 to 37 ), with zonal estimates ranging from 10 (Northern zone) to 37 (Coastal zone; table 1). Asking about MR increased abortion reporting as the estimate excluding MRs was 20 per 1000 women nationally (95\% CI: 16 to 25). However, we do not know what proportion of MRs would have been reported as abortions had the MR question not been asked first, so the estimate excluding MR should not be considered complete. For both the direct reporting and the confidante estimates, patterns across zones were similar, with rates in the Northern zone being significantly lower than in the Middle and Coastal zones.

The AICM yielded an abortion incidence rate of 27 per 1000 nationally (95\% CI: 22 to 32), with zonal estimates ranging from 19 (Northern zone) to 30 (Middle zone). ${ }^{2}$ 
Table 1 Abortion incidence rates from direct reporting (respondents) and confidante method, annualised over the past 3 years

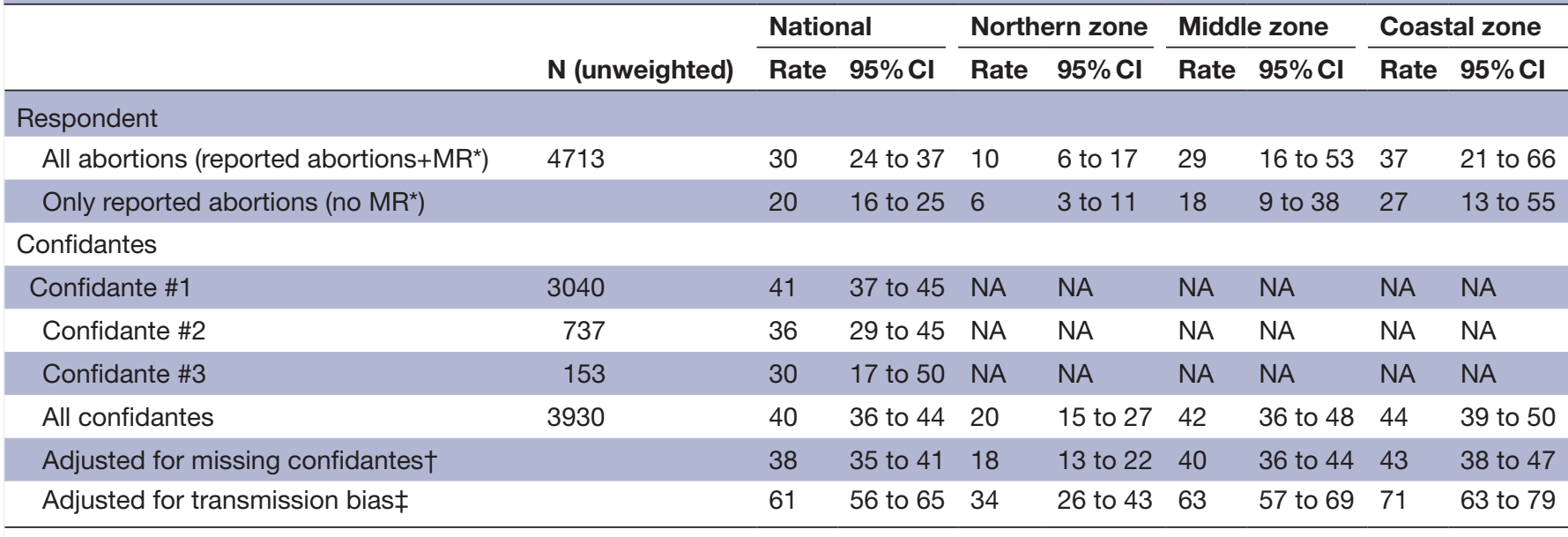

Northern zone includes Upper West, Upper East and Northern regions; Middle zone includes Brong-Ahafo, Ashanti, Eastern and Volta regions; Coastal zone includes Western, Central and Greater Accra regions.

*MR or action to bring back a late period without necessarily knowing whether one is pregnant.

†The $33 \%$ of respondents who reported no confidantes had different demographic characteristics and a lower abortion rate than those with at least one confidante. Assuming confidantes are similar to respondents on the aggregate, these 'missing' confidantes may have significantly different abortion behaviours than those in the data, which would affect the incidence rate. Thus, we predicted the likelihood of recent abortion for the missing confidante of each confidante-less respondent, using Poisson regression with the respondent's sociodemographic characteristics as covariates. ${ }^{29}{ }^{30}$ We then calculated the confidante abortion rates including the missing confidantes' data.

$\ddagger$ Adjusted for potential non-disclosure of some confidantes’ abortions to the respondent. Based on an assumption of reciprocity, such that confidante $\mathrm{X}$ is as likely to disclose her abortion to the respondent as the respondent is to disclose an abortion to confidante $\mathrm{X}$, we asked respondents who reported an abortion in the past 3 years whether they had disclosed it to each of their confidantes. We calculated the proportion of respondents who disclosed to each confidante $(1,2,3)$, took the inverse of this proportion as the transmission bias factor, computed an average of all three factors (weighted on the number of confidantes) and applied it to the incidence rate adjusted for missing confidantes.

MR, menstrual regulation; NA, not applicable.

For the modified AICM (table 3), the national abortion rate was 41 per 1000 (95\% CI: 36 to 47); zonal rates were similar for the Northern zone (27) and Coastal zone (28) and significantly higher for the Middle zone (60).

We summarise abortion incidence rates for the confidante method, direct reports, AICM and modified AICM nationally and for each zone (table 4). We compared them using several criteria discussed at greater length elsewhere. ${ }^{3}$ Key decision points are summarised below.

We tested the accuracy of the direct report estimate by comparing the PAC treatment rate among respondents (number of treated abortion complications per 1000 women 15-49) with that based on HFS caseloads (assumed to be reliable, although they may be overestimated if some abortions were misclassified as PAC due to stigma). Respondent PAC treatment rates were lower than HFS treatment rates nationally and in the Middle and Northern zones (table 5). Although women may under-report untreated abortions, we assume they are less likely to under-report the proportion of abortions that get treated (the first component of calculating treatment rates). This suggests that the lower treatment rate in the CBS is due to respondents under-reporting their abortions (second component of calculating treatment rates) and not to an invalid multiplier (inverse of the proportion of abortions that get treated). In the Coastal zone, the respondent treatment rate is similar to the HFS rate, possibly because lower stigma in the capital city means less under-reporting of abortions in the CBS, as well as less misclassification of abortion as PAC in the HFS.

As explained in the methods section, for the confidante method to generate valid estimates, confidantes should

Table 2 Computation of abortion incidence using the List Experiment

\begin{tabular}{|c|c|c|c|c|}
\hline & \multicolumn{2}{|l|}{ List $\mathbf{A}$} & \multicolumn{2}{|l|}{ List B } \\
\hline & Estimate & $95 \% \mathrm{Cl}$ & Estimate & $95 \% \mathrm{Cl}$ \\
\hline Mean events in list with abortion & 1.391 & 1.314 to 1.468 & 1.474 & 1.391 to 1.556 \\
\hline Difference in means (row 1-row 2) and $\mathrm{Cl}$ for difference in means & 0.014 & 0.000 to 0.060 & 0.057 & 0.000 to 0.117 \\
\hline Abortion incidence per 1000 women & 14 & 0 to 60 & 57 & 0 to 117 \\
\hline
\end{tabular}


be selected independently of their likelihood of having had an abortion. In the phone follow-up of 406 randomly selected respondents enquiring about whether they were thinking about abortion when selecting confidantes, $10 \%$ said yes, and $39 \%$ of these specifically said they selected a confidante because she had had an abortion. We might expect most of this bias to operate in selection of the first confidante, translating to an overinflated abortion rate for the first confidante and a drop-off for confidantes 2 and 3 (although selection of confidantes 2 and 3 could also be subject to this bias). Rates did drop slightly from confidante \#1 (41 per 1000) to confidante \#2 (36) and confidante \#3 (30), but these non-significant differences (table 1) could be caused by more limited knowledge of confidantes' abortions as respondents were asked to name confidantes in order of closeness. The transmission bias adjustment attempts to address this attrition but could lead to overinflation if the first confidante rate is overestimated.

To further test the accuracy of the confidante estimate, we calculated the PAC treatment rate among confidantes (based on an unadjusted number of abortions and proportion treated in facilities for complications) and compared it with that from the HFS. However, this rests on the assumption that respondents know if and where confidantes' abortions were treated and should thus be interpreted cautiously. The confidante PAC treatment rate was much higher (9.6 vs 5.7 per 1000 women; table 5), suggesting (under the assumption that HFS caseloads are accurate) overestimation of either the confidante abortion rate or the proportion of abortions treated. Although respondents may be more likely to know about confidantes' treated abortions (resulting in an overestimate of the proportion that got treated), this would be accompanied by an underestimation of the overall abortion rate and would not affect the final PAC treatment rate. It is unlikely that respondents would accurately report confidantes' abortions and over-report the proportion treated, providing further tentative evidence that the confidante abortion rate may be overestimated.

Comparing the AICM versus the modified AICM requires consideration of the two multipliers: the KIS multiplier for the AICM and the CBS multiplier for the modified AICM. Having ascertained the validity of the CBS multiplier previously, we considered it more reliable than the KIS multiplier, as it is based on respondents' direct reports of their behaviour, versus KIS experts' informed opinions. Both multipliers may be underestimated if respondents were more likely to report (CBS) or know about (KIS) complicated abortions that received treatment. Although multipliers cannot be directly compared as the KIS multiplier only applies to illegal abortions, ${ }^{2}$ the fact that the CBS multiplier (7.3) is higher than the KIS multiplier (3.9) suggests less underreporting of untreated abortions in the CBS, and confers additional validity to the CBS multiplier. A similar pattern was found in a study in Burkina Faso. ${ }^{23}$ 
Table 4 Abortion incidence rates from direct reporting, confidante method, AICM and modified AICM

\begin{tabular}{|c|c|c|c|c|c|c|c|c|}
\hline & \multicolumn{2}{|l|}{ National } & \multicolumn{2}{|c|}{ Northern zone } & \multicolumn{2}{|c|}{ Middle zone } & \multicolumn{2}{|c|}{ Coastal zone } \\
\hline & Estimate & $95 \% \mathrm{Cl}$ & Estimate & $95 \% \mathrm{Cl}$ & Estimate & $95 \% \mathrm{Cl}$ & Estimate & $95 \% \mathrm{Cl}$ \\
\hline $\begin{array}{l}\text { Direct reporting } \\
\text { (abortions+MR }^{\star}, 3- \\
\text { year annualised) }\end{array}$ & 30 & 24 to 37 & 10 & 6 to 17 & 29 & 16 to 53 & 37 & 21 to 66 \\
\hline $\begin{array}{l}\text { Confidantes (3-year } \\
\text { annualised) } \dagger\end{array}$ & 61 & 56 to 65 & 34 & 26 to 43 & 63 & 57 to 69 & 71 & 63 to 79 \\
\hline AICM & 27 & 22 to 32 & 19 & 15 to 22 & 30 & 21 to 40 & 25 & 19 to 32 \\
\hline Modified AICM & 41 & 36 to 47 & 27 & 22 to 33 & 60 & 48 to 72 & 28 & 24 to 33 \\
\hline Final estimate & 44 & NA & 21 & NA & 54 & NA & 45 & NA \\
\hline
\end{tabular}

Northern zone includes Upper West, Upper East and Northern regions; Middle zone includes Brong-Ahafo, Ashanti, Eastern and Volta regions; Coastal zone includes Western, Central and Greater Accra regions.

*MR or action to bring back a late period without necessarily knowing whether one is pregnant.

†Adjusted for missing confidantes and transmission bias.

fFinal estimate is the mean of the estimates from the three most valid approaches: direct reporting, confidante method and modified AICM. Instead of giving $95 \% \mathrm{Cls}$ (which would risk overstating the certainty of this final estimate), we give lower and upper bounds based on the lowest and highest estimate (these are presented in the text).

AICM, Abortion Incidence Complications Method; MR, menstrual regulation.

Based on available evidence, the approaches that met the basic criteria for validity and reliability in Ghana were the direct reports, the confidante method, and the modified AICM. The data do not allow us to determine which of these rates is the most accurate as we are estimating a hidden behaviour for which there is no gold standard. We therefore provide a range for the true abortion rate, with the lowest and highest estimates from the three approaches (direct, modified AICM and confidante) as the lower and upper bounds. In cases where a final point estimate of abortion incidence in Ghana is needed for policy and programmatic purposes, we suggest using the mean of the estimates from the three approaches, which yields a national abortion incidence rate of 44 per 1000 (range: 30-61). Zonal rates are 24 (range: 10-34) for the
Northern zone, 51 (range: 29-63) for the Middle zone and 45 (range: 28-71) for the Coastal zone.

Based on these final abortion estimates, the estimated national pregnancy rate was 194 pregnancies per 1000 women aged 15-49 years, and the unintended pregnancy rate was 103 per 1000; this varied by zone (online supplementary appendix B, table B1). Of all pregnancies, an estimated $53 \%$ were unintended, and $23 \%$ ended in induced abortion (online supplementary appendix B, figure B1).

\section{DISCUSSION}

This study contributes significantly to the field of abortion incidence measurement-and sensitive behaviour

\begin{tabular}{|c|c|c|c|c|}
\hline & National & $\begin{array}{l}\text { Northern } \\
\text { zone }\end{array}$ & Middle zone & $\begin{array}{l}\text { Coastal } \\
\text { zone }\end{array}$ \\
\hline \multicolumn{5}{|l|}{ CBS respondents } \\
\hline Proportion of CBS respondents who had abortions in past 3 years & 0.072 & 0.028 & 0.069 & 0.090 \\
\hline Proportion of respondents' abortions treated in a facility for complications & 0.136 & 0.106 & 0.128 & 0.146 \\
\hline Post-abortion care treatment rate* for CBS & 3.3 & 1.0 & 2.9 & 4.4 \\
\hline \multicolumn{5}{|l|}{ CBS confidantes } \\
\hline Proportion of confidantes who had abortions in past 3 years & 0.120 & 0.070 & 0.140 & 0.145 \\
\hline Proportion of confidante abortions treated in a facility for complications & 0.241 & 0.333 & 0.293 & 0.170 \\
\hline Post-abortion care treatment rate ${ }^{*}$ for confidantes & 9.6 & 7.7 & 13.6 & 8.2 \\
\hline \multicolumn{5}{|l|}{ HFS } \\
\hline Post-abortion care treatment rate ${ }^{*}$ & 5.7 & 2.9 & 7.7 & 4.2 \\
\hline
\end{tabular}

Northern zone includes Upper West, Upper East and Northern regions; Middle zone includes Brong-Ahafo, Ashanti, Eastern and Volta regions; Coastal zone includes Western, Central and Greater Accra regions.

*No of women per 1000 who were treated in a facility for abortion complications.

CBS, community-based survey; HFS, health facilities survey. 
measurement more broadly—by providing a rigorous investigation of five methodologies using data collected contemporaneously. Our findings do not reveal one approach that performed best. Furthermore, the performance of the approaches we tested is likely dependent on contextual factors like culture, stigma, abortion legality and literacy, so the approaches may perform differently elsewhere. For example, in countries where women tend to share less intimate information with friends, the confidante method may be inappropriate. ${ }^{23}$ In countries where abortion laws are more restrictive and stigma is higher, direct reporting would likely perform poorly, but the confidante method may perform well if women rely on their social networks to learn about and access care. ${ }^{1923}$ In countries where women may not share much intimate information but where abortion is broadly legal and less stigmatised, direct reporting may perform better than network-based approaches, as in Rajasthan. ${ }^{24}$ In Ghana, abortion is broadly legal but stigma persists. ${ }^{26} 27$ Thus, our direct report estimate is likely an underestimate of the true abortion rate, despite being higher than the 2017 GMHS estimate of 13 per 1000 (based on the GMHS finding that $6.7 \%$ of women had abortions in the past 5 years, assuming rates remained constant over those 5 years and not accounting for multiple abortions to the same person). ${ }^{7}$ Asking respondents about bringing back their period increased reporting and asking about confidantes' abortions earlier in the survey may have helped respondents feel more comfortable disclosing their abortions. This may be a reason to ask about confidantes' abortions in surveys, even if not utilising the method to calculate incidence.

Ensuring that confidantes are selected independently of their probability of abortion may be difficult if abortion is mentioned in the informed consent or a preceding question. If priming is a concern, the best friend approach ${ }^{20}$ may be superior as identifying one's best friend is likely less subject to interviewer influence than identifying women 'with whom one shares confidential information'. The main advantage of the confidante method over the best friend approach is the larger sample of confidantes. In Ghana, only $16 \%$ of women had more than one confidante; implementing the best friend approach would have reduced the confidante sample size from 3930 to 3040. Thus, the suitability of each approach depends on the respondent sample size and the size of women's close networks; future studies might conduct formative research to determine women's average number of confidantes prior to survey design.

The failure of the List Experiment in Ghana seems primarily due to poor understanding of the questions or insufficient directions on how to respond as the practice list also yielded an inaccurate estimate. The List Experiment performance is also sensitive to the nature of the other list items, which should meet certain criteria. ${ }^{16}$ While we endeavoured to fulfil these criteria, it is possible that small deviations contributed to the inconsistency in rates between lists $\mathrm{A}$ and $\mathrm{B}$, although this would not explain negative rates. The list experiment performed poorly in other low-resource countries ${ }^{15}$ and may not be appropriate in settings with low numeracy. The Northern zone (where the negative rate was found) has the lowest female literacy rate in Ghana (35 per 1000 vs 55 nationally). ${ }^{28}$

Although the modified AICM multiplier appeared to be valid in this setting, in countries where abortion reporting is lower due to stigma or illegality, or if the sample size is smaller, the number of self-reported abortion complications may be too low to calculate robust multipliers, making this approach inappropriate. In Ghana, the legality of abortion may complicate the implementation of AICM-based approaches, since the HFS PAC count may include some legal abortions reported as PAC due to stigma.

This was the first study to test more than four approaches to measuring abortion incidence in one population. We cross-validated various components of the estimates against each other and proposed several improvements to survey instruments, such as asking about bringing back a period and preceding direct reporting with questions about confidantes. In terms of limitations, the confidante estimates do not take into account multiple abortions to one woman, which may have contributed to the observed decline in rates over time. Moreover, we did not explicitly ask respondents about MR among their confidantes, which further complicates the comparability of the confidante and direct reporting estimates. Although we made adjustments to confidante data to account for biases around knowledge transmission and confidante sample selection, the validity of our final estimates rely on these adjustments being accurate. Limitations of the AICM are discussed elsewhere. ${ }^{2}$

Other factors merit consideration in determining the best use of limited resources to measure abortion incidence. To compare estimates across diverse settings, it may be important to select methodologies that do not rely on extensive sharing of intimate information between friends, or that perform well in low-literacy contexts. Other considerations include the level of anonymity retained by respondents in high stigma contexts, cost relative to the desired precision of estimates, and the potential for collecting contextual information on abortion (which can be of great policy relevance). Ultimately, it might be appropriate to employ more than one methodology to measure abortion incidence. For example, the confidante method might help improve direct reporting, whereas direct reporting is required for the modified AICM and can provide contextual information on abortion, even if not used for incidence estimation. Budget allowing, triangulating findings from different methodologies may yield the most robust estimate of this hidden and difficult to measure behaviour.

\section{Author affiliations}

${ }^{1}$ Guttmacher Institute, New York, New York, USA 
${ }^{2}$ Department of Population, Family and Reproductive Health, School of Public Health, Kwame Nkrumah University of Science and Technology, Kumasi, Ghana ${ }^{3}$ Department of Epidemiology, Johns Hopkins University Bloomberg School of Public Health, Baltimore, Maryland, USA

${ }^{4}$ Department of Population, Family and Reproductive Health, Johns Hopkins Bloomberg School of Public Health, Baltimore, Maryland, USA

${ }^{5}$ Department of Epidemiology and Biostatistics, Kwame Nkrumah University of Science and Technology, Kumasi, Ashanti, Ghana

\section{Twitter Chelsea B Polis @cbpolis}

Acknowledgements The authors are grateful to our fielding team and all study participants. They are also grateful to Joe Flack for programming our questionnaires in ODK, Philicia Castillo for analytic support on the AICM component, and Marjorie Crowell and Ernest Ekutor for research assistance at various stages of the project. This study was made possible by UK Aid from the UK Government and a grant from the Dutch Ministry of Foreign Affairs.

Contributors Contributions to the AICM component of this study are detailed elsewhere. SCK, EO, CBP, SOB, EKN and RL-R: led the conceptualisation of the project. SCK, EO, DWC, RH, CBP and SOB: participated in developing aspects of the methodological approach used. SCK, DWC, RH and SOB: participated in programming and implementing computer code for the analysis. SCK, DWC and $\mathrm{RH}$ : verified the overall reproducibility of research outputs. SCK, DWC and RH: conducted the formal analysis. E0, EKN and RL-R: led the data collection efforts. SCK, EO, DWC, RH, CBP, SOB and EKN: provided resources in terms of study materials, contact with respondents, and computing resources. SCK, DWC, RH, SOB and EKN: performed data curation to manage and clean data. SCK wrote the original draft of the manuscript. SCK, EO, DWC, RH, CBP, SOB, EKN and RL-R: participated in reviewing and editing the draft. SCK, CBP, EO and SOB: had oversight and leadership responsibility for research activity planning and execution, including mentorship external to the core team. SCK, EO, DWC, RH, CBP, SOB and EKN: were responsible for managing and coordinating research activity planning and execution. SCK and CBP: were responsible for acquisition of the funding support for the project, leading to this publication.

Funding UK Aid from the UK Government, and Dutch Ministry of Foreign Affairs.

Disclaimer The views expressed are those of the authors and do not necessarily reflect the positions and policies of the donors.

Competing interests None declared.

Patient and public involvement Patients and/or the public were not involved in the design, or conduct, or reporting, or dissemination plans of this research.

Patient consent for publication Not required.

Provenance and peer review Not commissioned; externally peer reviewed.

Data availability statement Data are available upon request. De-identified versions of the raw Community-Based Survey, Health Facilities Survey and Knowledgeable Informants Survey datasets collected by the authors and used in this analysis are available from the Guttmacher Institute upon reasonable request to researchers who wish to use the data for scholarly analysis. To discuss obtaining copies of these datasets, please contact popcenter@guttmacher.org with the detailed protocol for your proposed study, and information about the funding and resources you have to carry out the study.

Open access This is an open access article distributed in accordance with the Creative Commons Attribution Non Commercial (CC BY-NC 4.0) license, which permits others to distribute, remix, adapt, build upon this work non-commercially, and license their derivative works on different terms, provided the original work is properly cited, appropriate credit is given, any changes made indicated, and the use is non-commercial. See: http://creativecommons.org/licenses/by-nc/4.0/.

ORCID iD

Chelsea B Polis http://orcid.org/0000-0002-1031-7074

\section{REFERENCES}

1 Remez L, Singh S, Introduction TA. In: methodologies for estimating abortion incidence and Abortion-Related morbidity: a review. New York and Paris: Guttmacher Institute and the International Union for the Scientific Study of Population, 2010.

2 Polis CB, Castillo PW, Otupiri E, et al. Estimating abortion incidence in Ghana (2017) using the abortion incidence complications methodology. In preparation 2019.
3 Sedgh G, Keogh SC. Novel approaches to estimating abortion incidence. Reprod Health 2019;16:16-44.

4 Ahiadeke C. Incidence of induced abortion in southern Ghana. Int Fam Plan Perspect 2001:27:96-101.

5 Adjei G, Enuameh Y, Asante KP, et al. Predictors of abortions in rural Ghana: a cross-sectional study. BMC Public Health 2015;15:202.

6 Ghana Statistical Service (GSS), Ghana Health Service (GHS), ICF International. Ghana demographic and health survey 2014. Accra, Ghana: GSS, GHS, ICF International, 2015. https://dhsprogram.com/ pubs/pdf/fr307/fr307.pdf

7 Ghana Statistical Service (GSS), Ghana Health Service (GHS), ICF. Ghana maternal health survey, 2017. Accra, Ghana: GSS, GHS, ICF, 2018. https://www.dhsprogram.com/pubs/pdf/FR340/FR340. pdf

8 Rossier C. Estimating induced abortion rates: a review. Stud Fam Plann 2003;34:87-102.

9 Lara D, Strickler J, Olavarrieta CD, et al. Measuring induced abortion in Mexico: a comparison of four methodologies. Sociological Methods \& Research 2004;32:529-58.

10 Juarez F, Cabigon J, Singh S. The Sealed Envelope Method of Estimating Induced Abortion: How Much of an Improvement? In: Singh S, Remez L, Tartaglione A, eds. Methodologies for estimating abortion incidence and Abortion-Related morbidity: a review. New York and Paris: Guttmacher Institute and International Union for the Scientific Study of Population, 2010: 107-24.

11 Singh S, Remez L, Tartaglione A. Methodologies for estimating abortion incidence and Abortion-Related morbidity: a review. New York and Paris: Guttmacher Institute and International Union for the Scientific Study of Population, 2010. http://www.guttmacher.org/ pubs/compilations/IUSSP/abortion-methodologies.pdf

12 Singh S, Juarez F, Prada E, et al. Estimating abortion incidence: assessment of a widely used indirect method. Popul Res Policy Rev 2019;38:429-58.

13 Singh S, Prada E, Juarez F. The Abortion Incidence Complications Method: A Quantitative Technique. In: Singh S, Remez L, Tartaglione A, eds. Methodologies for estimating abortion incidence and Abortion-Related morbidity: a review. New York and Paris: Guttmacher Institute and International Union for the Scientific Study of Population, 2010: 71-98.

14 Moseson H, Massaquoi M, Dehlendorf C, et al. Reducing underreporting of stigmatized health events using the list experiment: results from a randomized, population-based study of abortion in Liberia. Int J Epidemiol 2015;44:1951-8.

15 Bell SO, Bishai D. Can a list experiment improve validity of abortion measurement? Stud Fam Plann 2019;50:43-61.

16 Glynn AN. What can we learn with statistical truth serum? design and analysis of the list experiment. Public Opin Q 2013;77:159-72.

17 Moseson H, Treleaven E, Gerdts C, et al. The list experiment for measuring abortion: what we know and what we need. Stud Fam Plann 2017;48:397-405.

18 Ghofrani M, Asghari F, Kashanian M, et al. Prevalence of induced abortion in Iran: a comparison of two indirect estimation techniques. Int Perspect Sex Reprod Health 2018;44:73-9.

19 Rossier C. Measuring Abortion with the Anonymous Third Party Reporting Method. In: Singh S, Remez L, Tartaglione A, eds. Methodologies for estimating abortion incidence and AbortionRelated morbidity: a review. New York and Paris: Guttmacher Institute and International Union for the Scientific Study of Population, 2010.

20 Yeatman S, Trinitapoli J. Best-friend reports: a tool for measuring the prevalence of sensitive behaviors. Am J Public Health 2011:101:1666-7.

21 Rastegari A, Baneshi MR, Haji-Maghsoudi S, et al. Estimating the annual incidence of abortions in Iran applying a network scale-up approach. Iran Red Crescent Med J 2014;16:e15765.

22 Sully E, Giorgio M. Estimating abortion incidence using the network scale-up method. Kenya: Watamu, 2018.

23 Sedgh G, Rossier C, Kaboré I, et al. Estimating abortion incidence in Burkina Faso using two methodologies. Stud Fam Plann $2011: 42: 147-54$

24 Elul B. Anonymous third-party reporting of induced abortion: an experiment in Rajasthan, India. Boston, MA, 2004.

25 Cumming G, Finch S. Inference by eye: confidence intervals and how to read pictures of data. Am Psychol 2005;60:170-80.

26 Aniteye P, O'Brien B, Mayhew SH. Stigmatized by association: challenges for abortion service providers in Ghana. BMC Health Serv Res 2016;16:486.

27 Shellenberg KM, Hessini L, Levandowski BA. Developing a scale to measure stigmatizing attitudes and beliefs about women who have abortions: results from Ghana and Zambia. Women Health 2014:54:599-616. 
28 Ghana Statistical Service (GSS), Ghana Health Service (GHS), ICF. Ghana malaria indicator survey 2016. Accra, Ghana and Rockville, Maryland, USA: GSS, GHS, ICF, 2017. https://dhsprogram.com/ pubs/pdf/MIS26/MIS26.pd
29 Bell S, Shankar M, Omoluabi EF, et al. Methodological Advances in Survey-Based Abortion Measurement: Promising Findings From Nigeria, India, and Cote d'Ivoire. Austin, Texas, 2019.

30 Gakidou E, King G. Death by survey: estimating adult mortality without selection bias from sibling survival data. Demography 2006;43:569-85 